\title{
Research and Progress on Virtual Cloud Laboratory
}

\author{
Jian Wei ZHANG ${ }^{2}$, Zhi Hui SHANG ${ }^{1}$, Chen YUAN ${ }^{1}$, Lin Lin MA ${ }^{1}$, Zeng Yu CAI ${ }^{1}$, Chun Hui HU ${ }^{2}$ \\ ${ }^{1}$ School of Computer and Communication Engineering, Zhengzhou University of Light Industry, Zhengzhou 450002, China \\ ${ }^{2}$ Software Engineering College, Zhengzhou University of Light Industry, Zhengzhou 450002, China
}

\begin{abstract}
In recent years, cloud computing technology has experienced continuous development and improvement, and has gradually expanded to the education sector. First, this paper will introduce the background knowledge of the current virtual cloud laboratory; by comparing the advantages and disadvantages between traditional laboratory and virtual cloud laboratory, and comparing the application, advantages and disadvantages, and development trend of OpenStack technology and VMWare technology in safety, performance, design, function, use case, and value of virtual cloud laboratory, this paper concludes that application based on OpenStack virtual cloud laboratory in universities and research institutes and other departments is essential.
\end{abstract}

\section{Introduction}

Many universities worldwide have numerous high laboratory teaching resources but low resource-sharing capability, which leads to the problem that laboratory teaching resources in universities often require multiple configurations to meet the need of laboratory teaching, or teaching resources cannot be effectively shared, resulting in a serious waste of teaching resources. Application of the virtual cloud computing platform is of great significance, having a great impact on integrating the hardware and software laboratory teaching resources in universities and improving the automation, networking and digitization of teaching. Typical universities are as follows: South China University of Technology mainly focuses on the service end design of OpenStack system; Sun Yat-Sen University mainly pays attention to the application of OpenStack in virtual classroom, and the major of Beijing University of Posts and Telecommunications is the research on OpenStack resource monitoring technology. The main purpose of those researches is to carry out secondary development on OpenStack, so that it can serve the school's teaching and research. By comparing the advantages and disadvantages between traditional laboratory and virtual cloud laboratory, and comparing the application, advantages and disadvantages, and development trend of OpenStack technology and VMWare technology in safety, performance, design, function, use case, and value of virtual cloud laboratory, this paper concludes that application based on OpenStack virtual cloud laboratory in universities and research institutes and other departments is essential.

\section{Overview of Virtual Cloud Laboratory}

\footnotetext{
a Jian-wei ZHANG: 1126483520@qq.com
}

\subsection{Overview}

Cloud computing ${ }^{[1]}$ is based on the increase, use and delivery model of internet-related service, usually involving in providing dynamic, scalable and often virtualized resources via the Internet. The definition by National Institute of Standards and Technology (NIST) is widely accepted currently: cloud computing ${ }^{[2]}$ is a payper-use model that supplies available, convenient, and on-demand network access, and gets into the shared pool of configurable computing resources (resources include network, server, storage, application, service), these resources can be provided quickly, and simply needs little management effort or little interaction with the service provider.

Thus,cloud computing mainly uses a distributed manner to reallocate computing resources, and and make distributed nodes work together to as to achieve simultaneous working. So in accordance with the form of service, the cloud is divided into services of the following three levels ${ }^{[3-4]}$ : Infrastructure as a Service (IaaS), Platform as a Service (PaaS) and Software as a Service (SaaS), as shown in figure 1: 


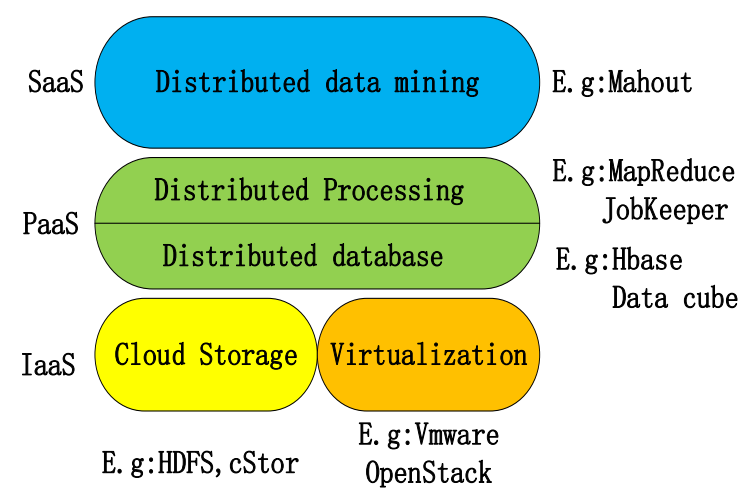

Figure 1. level of cloud computing services

Virtualization is to create a virtual version for some objects. It is an abstract method to represent computer resources. Through virtualization, abstracted resources can be accessed by the same way before the abstraction; a set of similar resources can be provided with a common abstract interface set, thereby hiding the differences between attribute and operation; a common way is allowed to view and maintain resources, including server virtualization, storage virtualization, application virtualization, platform virtualization, and desktop virtualization. Virtualization technology is a key technology that fully integrates and effectively uses a variety of computing and storage resources.

Cloud storage actually is also an application in teaching informatization cloud laboratory. For one thing, cloud storage can effectively arrange the teaching resources in laboratory so as to avoid the waste of teaching resources, and teachers and students can query related teaching resources through cloud storage. For another, cloud storage can integrate a variety of related teaching resources while investing in the construction of teaching resources, and provide multiple versions of teaching resources in different periods, so that teachers and students can make comparisons. Currently cloud storage technology in many colleges and universities has taken the lead, wherein the personal cloud storage services (jucbe) of Shanghai Jiaotong University run in 2012 has set us an example.

\subsection{Introduction of OpenStack architecture}

First, the role of each module in OpenStack architecture diagram will be introduced: the role of Heat is to arrange the required cloud user-defined OpenStack service, Horizon provides UI to other cloud services, Ceilometer is responsible for collecting the data of other services for monitoring, charges and other uses, another program that conducts authentication by Keystone to achieve the core of cloud computing is a virtual machine. In OpenStack, Nova is responsible for creating virtual machines, Glance provides mirror image for virtual machines, Cinder provides block storage for virtual machines, and Neutron is responsible for the network activity service of virtual machines. In addition, the object storage of OpenStack is in the charge of Swift, the image file of Glance and the backup volume of Cinder are stored on Swift. The overall architecture is shown in Figure 2:

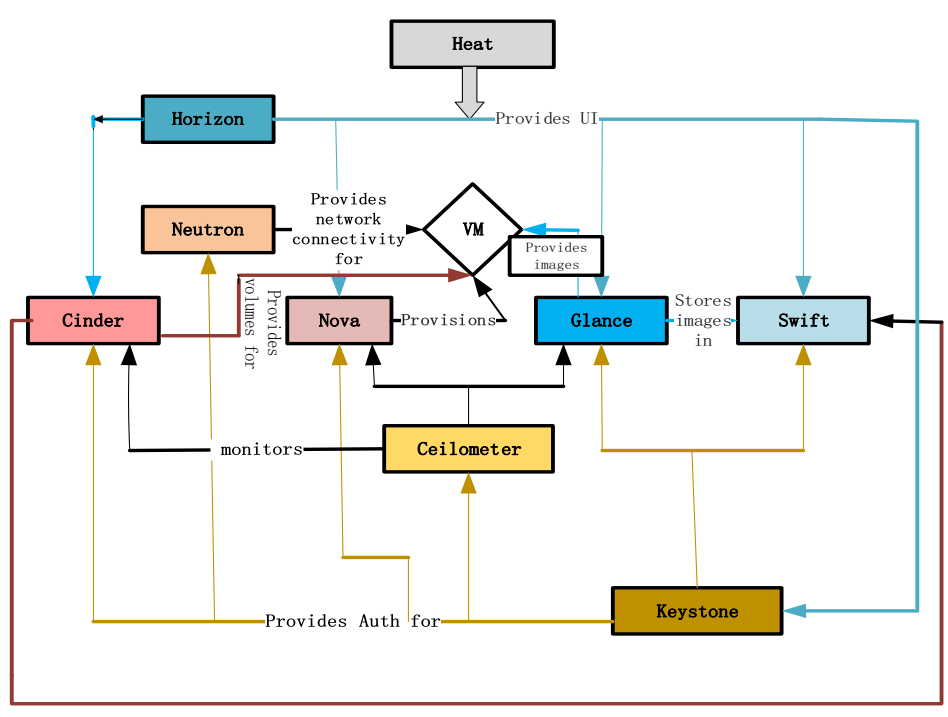

Figure 2. Architecture of OpenStack components

\section{Classification of Laboratory}

\subsection{Traditional Laboratory}

For the first time, Colleges and universities make a breakthrough in laboratory informatization teaching, which is a huge progress of the education sector. It is convenient for practical management and maintenance, as well as observation of failures and other problems. But with the gradual expansion of the scale of computer laboratory in universities, computer ownership is also on the increase. Many universities have the problem of large number of PC, high energy consumption, low resource utilization, management and maintenance difficulties and other issues, specifically in the six areas: tense funding, high energy consumption, difficult management and maintenance, low efficiency, low resource sharing, and difficult software update.

\subsection{Virtual Cloud Laboratory}

We will use cloud computing technology to build a private cloud of laboratory based on existing infrastructure, take advantage of virtualization to simulate rare hardware, build required experimental simulation environment, establish greater teaching and research platform, and provide large-scale reliable, scalable environment for software. In laboratory construction and management, laboratory built with the virtual cloud technology has the advantages that a traditional laboratory does not: less investment, high efficiency, high utilization, high experiment resource sharing, and convenient, fast and efficient management and maintenance of laboratory.

Compared with conventional laboratory, although virtual cloud laboratory boosts incalculable advantage, it also has its own drawbacks and shortcomings: Firstly, it 
has difficulties in management and maintenance; different from the traditional laboratory management, all the resources of virtual cloud computer laboratory is virtualized on the server, and configuration and management of virtual resources are relatively difficult, therefore, familiarity of management personnel with virtualization and cloud computing technology determines the management and maintenance effectiveness of virtual computer laboratory. Secondly, the three-dimensional image processing capacity is limited; three-dimensional image processing capability of virtualized server hardware resources is poor; professional 3D graphic image testing software 3Dmark is applied for testing; a CPU-intensive hardware can only bring $30 \%$ of the performance into play, thus, it can be seen that a virtual computer lab cannot complete experiments which require a lot of three-dimensional image processing such as 3Dmax, AutoCAD and other three-dimensional graphic softwares. Table 1 is a comparison of the advantages and disadvantages between traditional laboratory and virtual cloud laboratory.

Table 1. comparison of the advantages and disadvantages between traditional laboratory and virtual cloud laboratory

\begin{tabular}{|l|l|l|}
\hline Advantages & \multicolumn{1}{|c|}{$\begin{array}{c}\text { Traditional } \\
\text { Laboratory }\end{array}$} & $\begin{array}{l}\text { Virtual Cloud } \\
\text { Laboratory }\end{array}$ \\
\hline $\begin{array}{l}\text { For the first time, } \\
\text { informatization } \\
\text { teaching is achieved } \\
\text { which facilitates } \\
\text { effective management } \\
\text { and maintenance, as } \\
\text { well as observation of } \\
\text { failures and other } \\
\text { problems. }\end{array}$ & $\begin{array}{l}\text { Less investment, } \\
\text { high efficiency, } \\
\text { high utilization, } \\
\text { high experiment } \\
\text { resource sharing, } \\
\text { and convenient, } \\
\text { fast and efficient } \\
\text { management and } \\
\text { maintenance of } \\
\text { laboratory. }\end{array}$ \\
\hline Disadvantages & $\begin{array}{l}\text { Tense funding, high } \\
\text { energy consumption, } \\
\text { difficult management } \\
\text { and maintenance, low } \\
\text { efficiency, low } \\
\text { resource sharing, and } \\
\text { difficult software } \\
\text { update. }\end{array}$ & $\begin{array}{l}\text { Difficult } \\
\text { management and } \\
\text { maintenance, } \\
\text { limited three- } \\
\text { dimensional image } \\
\text { processing } \\
\text { capability, poor } \\
\text { three-dimensional } \\
\text { image processing } \\
\text { capability of } \\
\text { virtualized server } \\
\text { hardware resources. }\end{array}$ \\
\hline
\end{tabular}

\section{Development of Virtual Cloud Laboratory Technology}

In the cloud computing ecosystem, there are two types of users that need to use cloud computing resources: Traditional IT applications and Cloud-aware applications gradually rising in the tide of the Internet. The cloud computing Vision, function, feature comparison or trend of VMWare and OpenStack will give you a vivid portrayal. Applied architecture in the future should be treated the same as virtual machines: compared with cloud computing application pattern, "care" of VMware and various functions to protect the virtual machine become increasingly less important.

\subsection{Introduction of Virtual Cloud Laboratory based on OpenStack}

OpenStack [5] is a Apache-licensed free software and open source project co-developed and initiated by NASA and Rackspace, which is designed to provide software for the construction and management of public and private clouds. Private cloud of Rackspace based on OpenStack revenues $\$ 700$ million per year, an increase of more than $20 \%$. It creates an opportunity to enter the field of cloud computing for hosting providers who wish to provide customers with cloud services. The project can help enterprises and service providers to achieve the infrastructure services similar to Amazon EC2 and S3, and change the actual server resources into computing resources changing according to demand so as to provide services to users in the form of a virtual machine. In addition, it can also help companies or institutions to establish their own private cloud, integrate the internal computing resources to provide services for sectors. The main purpose of the current considerable research on OpenStack of domestic colleges and universities is to carry out secondary development, enabling it to serve the teaching and research of the school. Virtual cloud laboratory is to carry out secondary development through OpenStack open source so as to achieve the application in teaching.

Features:

(1)OpenStack is an open source project, which facilitates the development and utilization of virtual cloud laboratory.

(2)OpenStack private cloud business growth rate has been increased.

(3)Computing resources changing according to demand can provide services to users in the form of a virtual machine.

(4)Pace of cloud construction can be promoted; IT operating cost can be reduced; business agility can be increased, energy can be conserved, industrial upgrading can be promoted.

\subsection{Introduction of Virtual Cloud Laboratory based on VMW}

Virtual Machine ware is a "virtual PC" software company, and products of the company can provide solutions to server and desktop virtualization. Products of its virtualization platform include players; VMware life cycle management can provide control over the virtual environment, realizing multi-performance of computers. The method provided by VMware can speed up the transition to cloud computing while retaining existing investments and improving security and control.

It allows you to run two or more Windows, DOS, LINUX systems simultaneously on a single machine. Compared with the "multi-boot" system, VMWare uses a completely different concept. Multi-boot system can only run one system at a time and should re-start the machine when one system switches into another. VMWare really runs "simultaneously"; multiple operating systems can switch into each other on the platform of the main system, like standard Windows application. Each operating 
system can have a virtual partition and configuration without affecting the real hard drive data, and several virtual machines can be connected into a local area network with the network cards, which is extremely convenient. Performance of the operating system on VMware is lower than that directly on the hard disk, therefore, it is more suitable for learning and testing. Features:

(1) providing solutions to server and desktop virtualization.

(2)accelerating the transition to cloud computing.

(3) conducting virtual partition and configuration.

(4) starting multiple systems simultaneously.

\subsection{Contrast between OpenStack technology and VMware technology}

In Cloud area,contrast between OpenStack and VMware is discuss most. The contrast content can be completed by comparing the key point of the two cloud computing products in the data center application. The contrast content specifically includes the following six sections: safety, performance, design, function, customer use case and value.

\subsubsection{From the point of view of security}

The openness of OpenStack open source cloud platform allows us to quickly identify and fix security vulnerabilities, which can reduce the possibility of overflow, and has flexibility and compatibility. Generally speaking. open source project has a good track record, but they also have holes and vulnerabilities, OpenStack is still an emerging area. VMware has high safety, which can be viewed as two separate computers to use. If the network in virtual machines is set only for the host, external network can not access the internal network of the virtual machine.

\subsubsection{From the point of view of performance}

OpenStack lacks practicality. Currently most scientific research institutes, businesses and other organizations are using its open source for secondary development, so many of the functions need to perfect himself. However, the code is more flexible and has strong compatibility. VMware performance is not high, and support of some hardware is not good, which is mainly shown in some peripherals linked in the virtual machine. CPU performance of VMware virtual system have some losses compared with the real system, but the performance has not been not significantly attenuated. Performance of register operation, integer operation, floating-point operation and many other operations are varied compared with that of the real system.

\subsubsection{From the point of view of design}

OpenStack is an open source system, and no one single company is in control of the development path of
OpenStack. Many companies are supporting the development of OpenStack, because it has great market power, and the development of OpenStack is diversified. However, some problems have occurred; implementation and maintenance of OpenStack deployment and infrastructure costs much more than those of VMware. At the same time, due to the relatively fast pace of version updates, technical support document cannot keep up the pace.

The advantages of VMware product is in the robustness of its architecture, and users of high-profile have used it in a multi-scale data center environment. The drawback of VMware is that the software system is closed, and software development route is in full compliance with VMware's own development goals; users or consumers do not have any control in this regard.

\subsubsection{From the functional point of view}

Among OpenStack, KVM supports block storage migration, and dynamic migration of KVM allows a virtual machine to migrate from one virtual machine manager to another. OpenStack scheduler includes scheduling for the compute and volume; through a series of rule parameters and filters set by the administrator, OpenStack scheduler deploys a virtual machines to an appropriate host. In terms of filter, the scheduler is very flexible; users can complete a filter with JSON format themselves and the filter also contains a number of predefined filters. Although OpenStack scheduler is very flexible, it still cannot completely replace DRS.

VMware fault tolerant mechanism monitors the state of the virtual machine and all changes, these changes will be synchronized to the second backup ESX (i) server. The concept of fault tolerance is that whether it is the main host or the minor host that has a problem, as long as one can work, the virtual machine on the host maintains normal operation. VMware already does not require shared storage to achieve dynamic migration. When a virtual machine is migrated from one host to another, the virtual machine's memory status and data migration should be synchronized. If the case is a shared memory, in fact, the data does not need to be migrated, only the link pointing to the data storage should be changed. This can reduce the network load during replication while accelerating the migration speed.

\subsubsection{From the functional point of use case}

Cloud computing application is not required. When a virtual machine has a problem, a new virtual machine will replace it. OpenStack inherited this advantage. Although VMware has many functions that OpenStack does not, for cloud computing applications, these functions become less important. In future, you probably will pay for the uncontrollable additional functions of VMware, which is of little use. Compared with cloud computing applications, the maintenance of VMware, and the protection of the various functions of virtual machines become increasingly less important. 


\subsubsection{From the functional point of value}

It is still not clear whether OpenStack is more valuable than VMware, and the answer depends on the size of the deployment. Although OpenStack is free to use, it needs numerous engineering resources and experts, as well as a lot of effort in architecture and construction. As it supports many deployment scenarios, and the installation processes are different. VMware needs some funds to purchase rights, and is relatively easier to install and run; in addition, compared with the command line, the learning cost of VMware is lower.

Generally speaking, OpenStack has high barrier to entry, but with the expansion of the scale of the project, you will benefit from it, because you do not need to pay high copyright fees. Although VMware is relatively easy to install in a small scale, but with the expansion of the scale, things have changed. That is, with the large-scale expansion of cloud application, we are more familiar with OpenStack, so barrier to entry into OpenStack is much lower. Not long ago, the CEO of OpenStack Foundation, Jonathan Bryce, said at the OpenStack summit in Vancouver, Canada that it was OpenStack that made the software more meaningful and things became more modular and easier with OpenStack. At the same time, he also clearly showed that the deep integration of that OpenStack community and the Docker community has entered a substantive stage, which is the direction of future development.

\subsubsection{Comparison Results between OpenStack and VMware Shown in the Figure}

Thus, VMware is better than OpenStack in safety, design and function, but worse than it in performance, use case and value. If the full point is 10 in safety, performance, design and other aspects, then the gap among those comparison trend can be seen in the bar chart, as well as the comprehensive comparison trend between VMware and OpenStack. The comprehensive comparison between VMware and OpenStack is shown in Figure 3.

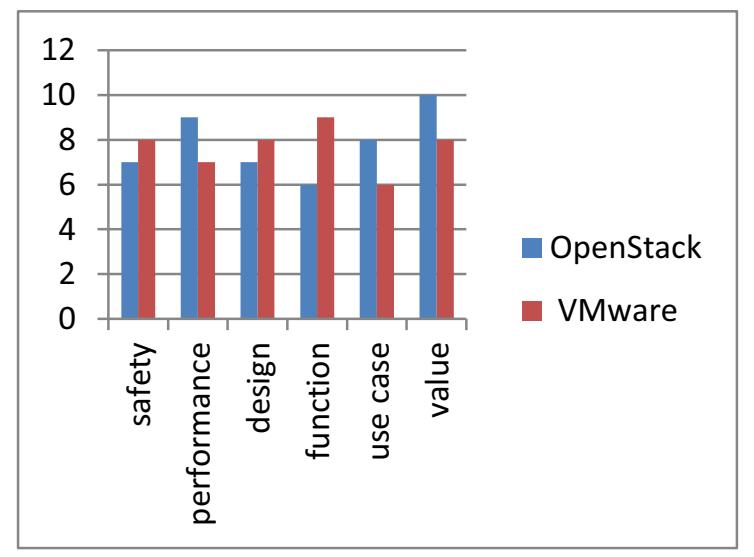

Figure 3. The comprehensive comparison chart between OpenStack and VMware
1 OpenStack technology takes an advantage over VMware technology in overall.

2 Virtual cloud laboratory based on OpenStack technology is the future development direction of the laboratory.

\section{Conclusion}

In summary, through the discussion of cloud computing, cloud storage, and virtual cloud of virtual cloud laboratory, the advantages and disadvantages of traditional laboratory and virtual cloud laboratory can be found, and finally we come to the conclusion that virtual cloud lab will be the development trend of laboratory in the information age. Through the comparison between OpenStack technology and VMware technology, the advantages of OpenStack technology in virtual cloud laboratories can be seen. Application of virtual cloud platform based on OpenStack technology will be the development direction in the next few years, and it can further improve the digitalization, networking, and automation of virtual experiment teaching.

\section{Acknowledgements}

This work is Supported by 2016 Outstanding Innovation Science and Technology Youth Project of the Innovation Scientists and Technicians Plan of Henan Province: research of the macro scenario fitting routing technology centering on content. Zhengzhou 2015 science and technology development plan (key science and technology): research on virtual cloud desktop scheduling key technology based on OpenStack (153PKJGG26). the Graduate's Scientific Research Foundation of Zhengzhou University of Light Industry.

\section{References}

1. Zhang Q,Cheng L,Boutaba R.Cloud computing: state-of-the-art and research challenges[J]. Journal of internet services and applications, vol.1,no.1, 7-18 (2010).

2. Armbrust M, Fox A, Griffith R, et al. A view of cloud computing $[\mathrm{J}]$. Communications of the ACM, vol.53,no.4, 50-58(2010).

3. Mell P, Grance T. The NIST Definition of Cloud Computing[J]. NIST Special Publication,145(2011).

4. Luo J Z,Jin J H, Song A B, et al. Cloud computing: architecture and key technologies[J].Journal of China Institute of Communications, vol.32,no.7,3-21(2011).

5. OpenStack Cloud Administrator Guide[J]. docs.openstack.org, (2014).

6. Cao Wei. Application of cloud computing platform based on OpenStack in university teaching. Hei Longjiang Information Technology,vol..26,183184(2014).

7. Wan Hongfeng. Construction and application of teaching cloud computing platform based on

Conclusion: 
OpenStack.China High Technology Enterprises,No.20,45-46(2015).

8. Lv Site. Virtualization, A Beginner's Guide. Bei Jing: China Machine Press, (2011).

9. Ren $\mathrm{Yu}, \mathrm{Li}$ Qingrong. Research on virtualization resource management platform based on VMware vSphere. Computer Applications and Software.vol.5,219-223(2012).

10. Sun Tao, Qiao Zhihe, Li Juan. Application of virtual cloud technology in computer laboratory construction management. Journal of Daqing Normal University,vol33,no.3,139-141(2013).

11. Zhang Jianwei, Wang Lu, Cai Zengyu,et al. System design and implementation of college graduation project whole-process management based on Web. Journal of Zhengzhou University of Light Industry: natural science edition,vol.24,no.5,54-58(2009).

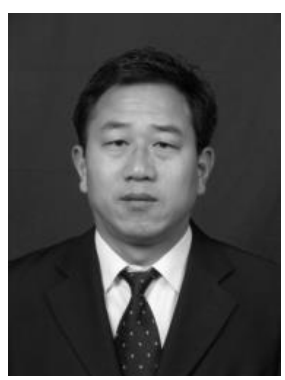

Jian-wei ZHANG,born in April, 1971, Henan, P R china Current position, grades: Professor at Zhengzhou University of Light Industry, China University studies: $\mathrm{PhD}$ degree from The PLA Information Engineering University in China Scientific interest: broadband information network and network security .

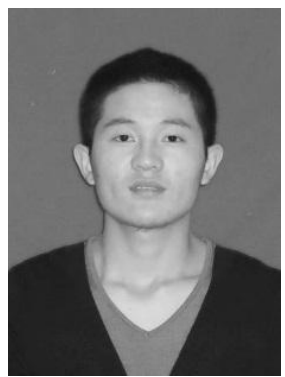

Zhi-hui SHANG, born in November, 1989, Henan, grades: A graduate student at Zhengzhou University of Light Industry, Scientific interest: Cloud computing and computer network . 